\title{
GABAergic Inhibition Controls Neural Gain in Inferior Colliculus Neurons Sensitive to Interaural Time Differences
}

\author{
Neil J. Ingham and David McAlpine \\ The Ear Institute and Department of Physiology, University College London, London WC1E 6BT, United Kingdom
}

We investigated the role of GABAergic inhibition on the responses of inferior colliculus (IC) neurons sensitive to interaural time differences (ITDs) in anesthetized guinea pigs. Responses to static and dynamic ITDs were obtained before, during, and after recovery from ionotophoretic application of $\mathrm{GABA}$, or antagonists to the $\mathrm{GABA}_{\mathrm{A}}$ receptor gabazine and bicuculline. For most neurons, a linear relationship was observed between discharge rates evoked by a particular ITD during drug application and control discharge rates. Blocking GABAergic inhibition, or adding exogenous GABA, scaled IC discharge rates in a multiplicative (divisive) and/or additive (subtractive) manner. When the influence of iontophoresed GABA antagonists or exogenous GABA on discharge rates was accounted for, GABAergic inhibition was found to have no effect on the ITD tuning properties of IC neurons. The tuning sharpness of ITD functions, the ITD that evoked $50 \%$ response magnitude, and the relative symmetry of ITD functions around their peak response were unaffected by blockade of inhibition or addition of tonic inhibition. However, the ability of neurons to discriminate between ITDs by virtue of differences in their discharge rate was altered by blocking or adding GABA. We propose that inhibition in the IC is involved in the control of the neural gain of the output of IC neurons rather than the regulation of ITD tuning. This gain control appears to arise from a combination of additive and multiplicative processes, and may involve mechanisms such as shunting inhibition or changes in the efficacy of inhibitory and excitatory inputs.

Key words: inferior colliculus; inhibition; GABAergic; interaural time differences; gain control; iontophoresis

\section{Introduction}

The inferior colliculus (IC), the major auditory nucleus of the midbrain, receives input from numerous ascending pathways in the auditory brainstem (Beyerl, 1978; Adams, 1979, 1980; Andersen et al., 1980; Oliver, 1987; Friauf and Kandler, 1990; Oliver et al., 1995, 2003; Takahashi and Konishi, 1988; Winer et al., 2002). Although many basic responses of IC neurons have been characterized (Irvine, 1992), specific roles for the IC in auditory processing remain to be determined. Because the IC lies downstream of brainstem neurons that process binaural spatial cues, a number of studies have investigated the way in which neural processing of such cues is transformed between the brainstem and the IC. Several studies postulate neural tuning for interaural time differences (ITDs) might be sharpened (Fujita and Konishi, 1991; Fitzpatrick et al., 1997; Spitzer and Semple, 1998; Sterbing et al., 2005), or that a transformation in neural coding for spatial hearing occurs from the "cues-based" strategy observed in the brainstem to a "context-based" strategy (Spitzer and Semple, 1991, 1995, 1998; Sanes et al., 1998; McAlpine et al., 2000). In both cases, a role for GABAergic inhibition has been

\footnotetext{
Received Jan. 12, 2005; revised May 18, 2005; accepted May 18, 2005.

This work was supported by a Medical Research Council Career Establishment Grant to D.M.

Correspondence should be addressed to Dr. David McAlpine, The Ear Institute and Department of Physiology, University College London, Gower Street, London WC1E 6BT, UK. E-mail: d.mcalpine@ucl.ac.uk.

N. J. Ingham's present address: Centre for the Neural Basis of Hearing, Physiological Laboratory, Downing Street, Cambridge CB2 3EG, UK.

D01:10.1523/JNEUROSCI.0146-05.2005

Copyright $\odot 2005$ Society for Neuroscience $\quad$ 0270-6474/05/256187-12\$15.00/0
}

postulated. The IC receives significant GABAergic innervation (Oliver and Beckius, 1992; Caspary et al., 1995; GonzalezHernandez et al., 1996; Burger and Pollak, 1998, 2001; Zhang et al., 1998; Casseday et al., 2000), including bilaterally from the dorsal nucleus of the lateral lemniscus (DNLL) (Adams and Mugnaini, 1984). The IC also has an extensive population of GABAergic neurons (Brunso-Bechtold et al., 1981; Oliver et al., 1994), and blocking GABAergic inhibition locally in the IC alters aspects of temporal and frequency response properties of IC neurons (LeBeau et al., 1996, 2001).

Although it is likely that GABA contributes to processing of specific acoustic tasks, including binaural cues, an intriguing possibility is that GABA has a more general effect on IC responses, that of neural gain control-a role only hinted at previously (Fujita and Konishi, 1991; Pollak and Park, 1993). Gain control, an important feature of any sensory systems, enables neurons to adjust the limited range of their firing rates to their input (Ohzawa et al., 1982, 1985; Chance et al., 2002; Mitchell and Silver, 2003). We tested the hypothesis that the IC acts as a site for controlling neural gain. We demonstrate that for individual neurons, blocking GABAergic inhibition or adding exogenous GABA modifies the discharge rate at each ITD by a common ratio, a common offset, or a combination of both. It does so without altering neural tuning for preferred ITDs or sharpening ITD tuning, suggesting that these proposed functions of GABAergic in the IC do not occur. Nevertheless, modulating the discharge rate by blocking GABAergic inhibition or adding exogenous GABA does alter the ability of single neurons to discriminate between 
different ITDs by virtue of differences in their discharge rate as the discharge rate increases or decreases. This is consistent with the proposed function of the IC as a site for neural gain control.

\section{Materials and Methods}

Many of the procedures used in this study have been published previously (McAlpine et al., 2000; McAlpine and Palmer, 2002) and will be outlined briefly here.

Preparation and recording. Single-unit recordings were made from the right IC of guinea pigs anesthetized with urethane $(1 \mathrm{~g} / \mathrm{kg}$ of a $20 \%$ solution). Additional analgesia was obtained using Hypnorm $(1 \mathrm{ml} / \mathrm{kg}$; fentanyl citrate/fluanisone) and additional doses administered as required. Atropine sulfate $(0.06 \mathrm{mg})$ was administered to reduce bronchial secretions. A tracheal cannula was inserted and core body temperature $\left(37^{\circ} \mathrm{C}\right)$ was maintained using a thermostatically controlled heating blanket and rectal probe. Animals were mounted in hollow ear speculas in a modified stereotaxic frame. A craniotomy was performed to allow microelectrode access to the right IC. Microelectrodes were mounted on a piezoelectric stepper motor (Inchworm IW-700/710; Burleigh Instruments, Victor, NY) for stereotaxic insertion into the IC. Parylene-coated tungsten microelectrodes (1-5 M $\Omega$; World Precision Instruments, Sarasota, FL) were used to define IC regions containing low-characteristic frequency (CF), ITD-sensitive neurons.

Iontophoresis and drugs. Glass pipette barrels of Carbostar- 4 microiontophoresis electrodes (Kation Scientific, Minneapolis, MN) were backfilled with drug solutions. GABA receptors on IC neurons were targeted. The antagonists GABAzine (SR95531 [2-(3-carboxypropyl)-3-amino-6(4-methoxyphenyl)-pyridazinium bromide] hydrobromide; $25 \mathrm{~mm} ; \mathrm{pH}$ 3.5; Tocris, Bristol, UK) or bicuculline methiodide (5 mm; pH 3.5; Sigma, St. Louis, MO) and agonist GABA (500 mm; pH 3.5, Sigma) in distilled water, were used. The remaining iontophoresis barrel was filled with $0.5 \mathrm{~m} \mathrm{NaCl}, \mathrm{pH} 3.5$, for current balancing. Retention and ejection currents were applied to the drug barrels via a Neurophore BH2 iontophoresis system using IP2 current pumps (Digitimer, Herfordshire, UK). A glass-coated carbon fiber served as the recording electrode $(\sim 0.5 \mathrm{M} \Omega$ impedance). The Carbostar- 4 replaced the tungsten electrodes on the Inchworm and was returned to the same stereotaxic coordinates.

Drugs were retained in the pipette barrels with a negative holding current of 10-20 nA. Drugs were ejected by the application of a positive ejection current (typically $<100 \mathrm{nA}$ ) to the pipette barrels. Controls were performed, in some cases, by an equivalent current injection from the balance pipette. No changes in response were noted. In other cases, the application of both antagonists and agonists of GABA receptors to the same neuron increased and decreased discharge rates, respectively, despite the different drugs having the same positive ejection currents.

Neural signals detected by the microelectrodes were filtered and amplified via a RA16 Medusa system (Tucker-Davis Technologies, Alachua, FL). Discriminated spikes were time stamped with $1 \mu$ s accuracy (Tucker-Davis Technologies; event timer ET1 and signal discriminator SD1) and recorded using integrated stimulus presentation and data acquisition software (developed by T. M. Shackleton and A. R. Palmer, Medical Research Council Institute of Hearing Research, Nottingham, UK).

Stimulus production and presentation. Acoustic stimuli were presented independently to each ear via custom-modified Beyerdynamic DT48 drivers coupled to damped 4-mm-diameter tubes and sealed into the hollow ear bars. Probe tube microphones (modified FG3452; Knowles Electronics, Burgess Hill, UK), inserted to within a few millimeters of the tympanic membrane, were used to calibrate the sealed acoustic delivery system in decibels, with respect to $20 \mu \mathrm{Pa}$. The sound systems for each ear were flat to within $\pm 3 \mathrm{~dB}$ from $50-2000 \mathrm{~Hz}$ and were matched to within $\pm 3 \mathrm{~dB}$ for this range.

All stimuli were generated at $100 \mathrm{kHz}$ sampling rate (AP2 array processor; Tucker-Davis Technologies), filtered (low pass; fc, 40 kHz; FT6; Tucker-Davis Technologies) and attenuated (PA4; Tucker-Davis Technologies) to the desired level. Search stimuli consisted of $50 \mathrm{~ms}$ pure tones of variable frequency and intensity, presented with zero interaural delay. When a single neuron was isolated, its CF and threshold were determined audiovisually. In most cases, a frequency-versus-level response area was mapped for frequencies two octaves above and four octaves below $\mathrm{CF}$, in $5 \mathrm{~dB}$ steps over a range of $\sim 0-80 \mathrm{~dB}$ sound pressure level. A range of interaurally delayed stimuli were presented, at $20 \mathrm{~dB}$ above the threshold of a neuron for the zero-delayed stimulus.

Tone bursts ( $50 \mathrm{~ms}$ duration; $2 \mathrm{~ms}$ rise-fall time) with ITDs over the range equivalent to \pm 1.5 cycles of the stimulus period were presented in a random order and repeated 10 times. Neural responses were plotted online as a function of ITD to generate tone delay functions (TDFs).

Noise bursts ( 50 ms duration; $2 \mathrm{~ms}$ rise-fall time; bandwidth, $50 \mathrm{~Hz}$ to $5 \mathrm{kHz}$ ) with ITDs over the range equivalent to \pm 1.5 cycles of the neural $\mathrm{CF}$ were presented in a random order and repeated 10 times. Neural responses were plotted online as a function of ITD to generate noise delay functions (NDFs).

Binaural beat stimuli were generated by the presentation of $3 \mathrm{~s}$ duration pure tones ( $2 \mathrm{~ms}$ rise-fall time) with a $1 \mathrm{~Hz}$ frequency difference between the ears. This produces a stimulus in which interaural phase difference (IPD) is linearly modulated over a complete cycle of IPD at a rate of once per second, such that the entire IPD range is traversed three times during the $3 \mathrm{~s}$ of the stimulus. Responses were recorded to 10 presentations of the binaural beat stimuli. Averaged neural responses were plotted online as a function of IPD for the central two cycles of the binaural beat to generate beat-IPD functions.

Data analysis. For complete analysis, it was critical that responses were recorded under control conditions, during iontophoresis of drug, and on full recovery of control responses after termination of the drug ejection current. Responses (spike rate; hertz) were plotted with respect to ITD, and with respect to the equivalent IPD of the stimulus frequency (for pure tones) or with respect to the neural CF (for noise) over \pm 0.5 cycles. Therefore, because all interaurally delayed stimuli contained ongoing delays only, and no onset delays, the terms "ITD" and "IPD" are used interchangeably. Functions were smoothed, such that each point represents the average discharge rate of the particular IPD and immediately adjacent IPD responses.

Iontophoretic application of antagonist or agonist of GABA receptors elicited dramatic changes in stimulus-evoked discharge rate. Discharge rates evoked at each IPD for the drug and recovery conditions, when plotted as a function of discharge rates evoked under control condition to produce "gain functions," which can be described by simple linear regression. Data were fitted with a regression line, based on the function $y=m x+c$, where $x$ is the control spike rate, $y$ is the drug or recovery spike rate, $m$ is the function slope, $c$ is the $y$ intercept. To assess changes in IPD tuning during drug application, discharge rates were scaled according to the discharge rates evoked in the drug and recovery conditions. Each IPD function was scaled by subtracting the intercept $c$, calculated from the gain function, from the spike rate at each IPD, and dividing by the slope of the gain function, $m$. For conditions in which the discharge rate increased during GABA-blocked conditions, this does not simply remove off-peak spikes but scales these discharge rates toward control levels. GABA functions could not reasonably be scaled up to control function levels. In many of the GABA functions, particularly at higher GABA ejection currents, spike rate was zero. If such functions were scaled up, the function had a large flat baseline, with a small peak above, making calculation of $50 \%$ tuning width essentially meaningless. As such, to overcome this problem, control functions were scaled down to those obtained the GABA ejection current. This simple scaling procedure produced overlapping IPD functions from which estimations of changes in IPD tuning width were made.

Tuning of IPD functions was assessed by measurement of the function width at $50 \%$ of the scaled maximum. The $50 \%$ positions, in cycles of IPD, were also noted. Mean best phase [using the method of Goldberg and Brown (1969)] was calculated from nonscaled functions. ITD discrimination was assessed by measuring the standard separation $D$ (Green and Swets, 1966).

We assume that the spike rates measured under control conditions before drug application are proportional to the input current. Thus, from plots of spike frequency (under application of drug or recovery conditions) against a factor proportional to the input current (i.e., control spike rate), it is possible to assess the phenomenological properties of the 

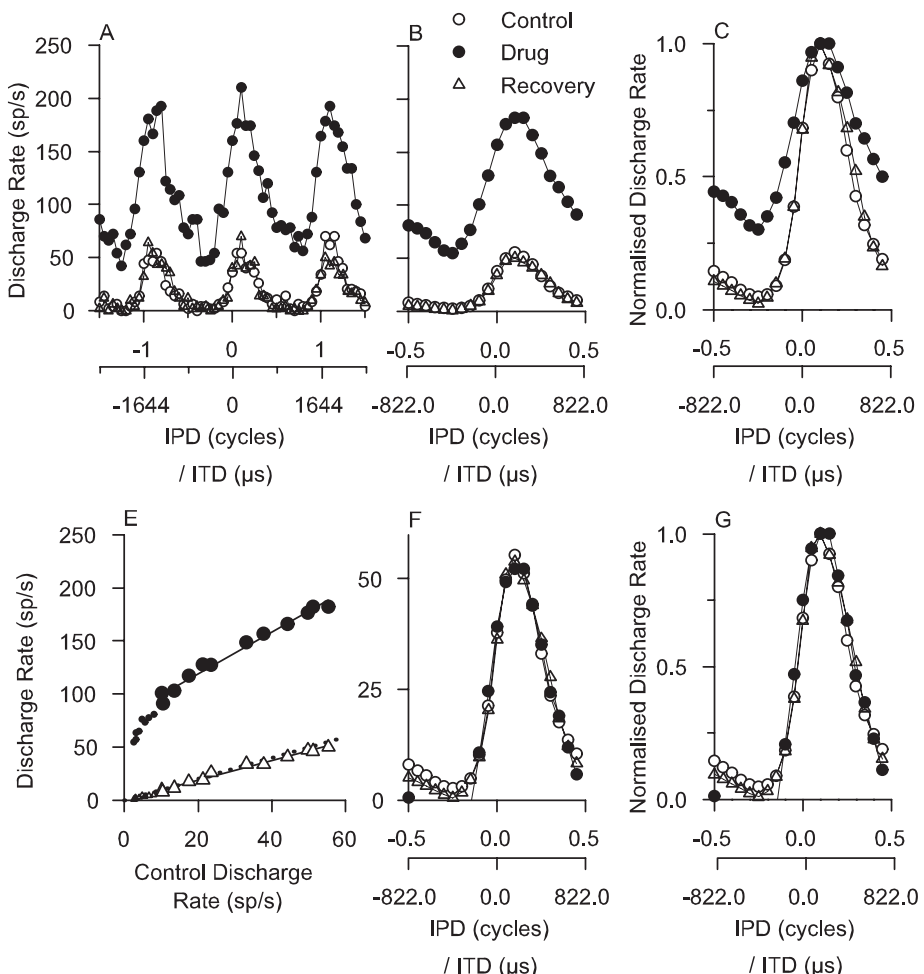

Figure 1. TDFs (spike rate vs ITD/IPD) recorded from a neuron before, during, and after recovery from the effects of iontophoresis of the GABA antagonist gabazine. Control, gabazine, and recovery responses are indicated by the key. $\boldsymbol{A}$, Raw TDFs recorded over a range of ITDs equivalent to \pm 1.5 cycles of the stimulus period of the carrier frequency ( $608 \mathrm{~Hz}$ in this case). $\boldsymbol{B}$, Smoothed and folded TDFs, plotted over \pm 0.5 cycles IPD. C, TDFs plotted normalized to the peak of the functions in $\boldsymbol{B}$. D, Fifty percent tuning width (cycles of IPD) of normalized IPD functions, recorded under control, drug, and recovery conditions. $E$, For each IPD configuration, discharge rates recorded under gabazine or recovery conditions are plotted as a function of those recorded under control conditions (gain functions). Linear regression lines fitted to each curve are shown. Dotted line indicates line of equality. $F$, TDFs scaled down to control levels, based on the regression parameters from $\boldsymbol{E}$. G, TDFs plotted normalized to the peak of the scaled functions in $\boldsymbol{F}$. $\boldsymbol{H}$, Fifty percent tuning width (cycles of IPD) of scaled and normalized IPD functions recorded under control, gabazine, and recovery conditions. sp, Spike; C, control; R, recovery.

control of neural gain in operation in low CF neurons in the IC. Linear regressions allow assessment of the "additive" (offset) and "multiplicative" (scaling) gain components (from the function intercept and slope, respectively) attributable to inhibitory mechanisms in the response of these neurons.

\section{Results}

Recordings were obtained from 20 low-CF $(<2 \mathrm{kHz})$, well isolated, ITD-sensitive single neurons from the right inferior colliculus of eight guinea pigs in response to interaurally delayed tones, interaurally delayed broadband noise, and binaural beats.

\section{Influence of GABAergic inhibition on pure-tone ITD sensitivity in IC neurons}

Figure $1 A$ shows the response of an IC neuron to interaurally delayed tones over ITDs in the range of $\pm 2467 \mu$ s, equivalent to \pm 1.5 cycles of the stimulus period ( $1645 \mu$ s for a carrier frequency of $608 \mathrm{~Hz}$, the CF of the neuron). ITD functions are shown for responses before $(\bigcirc)$, during $(\mathbf{O})$, and after recovery from the effects of $(\triangle)$ iontophoretic addition of the $G_{A B A}$ antagonist gabazine. The same data are shown in Figure $1 B$ but collapsed onto a single cycle of IPD. Blocking GABAergic inhibition with gabazine produced, maximally, a fourfold increase in peak discharge rate compared with predrug levels (Fig. 1A,B). When functions were normalized to their maximum discharge rate (Fig. 1C), ITD tuning at 50\% maximum response was

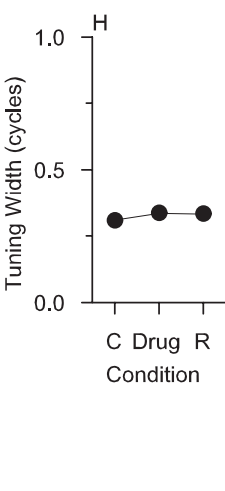

broader (Fig. $1 D$ ) —a wider range of ITDs evoked discharge rates of at least $50 \%$ of the maximum. This analysis suggests a role for GABAergic inhibition in sharpening the tuning of IC neurons for spatial cues, consistent with conclusions reached by others (Fujita and Konishi, 1991; Sterbing et al., 2005).

Figure 2 shows the response of an IC neuron to interaurally delayed tones before $(\bigcirc)$, during $(\mathbf{O})$, and after recovery from the effects of $(\triangle)$ iontophoresis of GABA itself (Fig. $2 A, B$ ). Iontophoresis of GABA had the opposite effect to iontophoresis of gabazine, with discharge rates being substantially reduced compared with control discharge rates. After normalization to maximum discharge rate, ITD tuning appeared to be sharpened (Fig. $2 C, D)$ compared with control responses.

The data described thus far suggest blocking GABAergic inhibition broadens neural tuning for ITD, whereas adding GABAergic inhibition sharpens such tuning. However, this conclusion depends on the normalization procedure applied. All normalization procedures have underlying mathematical assumptions as to the form of the processes responsible for generating and controlling spike output. In the normalization to maximum discharge rate described thus far and used previously in studies suggesting that ITD tuning is sharpened by GABAergic inhibition (Fujita and Konishi, 1991; Sterbing et al., 2005), these assumptions are unknown, and the processes are not quantified. However, by the assumption of two general and well established neural processes, additive and multiplicative gain control, it is possible to provide a satisfactory explanation of the responses of delay-sensitive IC neurons to changes in inhibitory input.

To apply the concept of neural gain to the responses of IC neurons, a simple procedure was developed to attempt an appropriate scaling of interaural delay functions, based on the relationship between the discharge rates evoked under control conditions and those evoked under drug and recovery conditions. For each neuron under conditions of GABA block or addition of exogenous GABA, discharge rates evoked at each ITD/IPD were plotted as a function of control discharge rates before application of drug. The resulting gain functions (Figs. $1 E, 2 E$ ) were used to quantify the extent to which multiplicative and additive mechanisms could account for any changes in the response.

The effect of blocking GABAergic inhibition on neural gain is illustrated in Figure $1 E$. Iontophoresis of gabazine increased the slope of the function describing the discharge rate during GABA block as a function of the discharge rate during control (predrug) conditions above unity (Fig. 1E, O) (slope, 1.96). Conversely, iontophoresis of exogenous GABA reduced the slope of the gain function to 0.39 (Fig. 2 E). Together, this suggests that GABAergic inhibition scales discharge rates multiplicatively (divisively), because the discharge rate was multiplied by a common factor when GABA was blocked-the slope of the gain function increased- 
and divided by a common factor when exogenous GABA was added-the slope of the gain function decreased.

In addition to a change in slope, there was also a change in the offset of the delay functions that applied at all IPDs. This was seen as an increase in the IPD-insensitive baseline discharge rate during GABA block (Fig. $1 A, B$ ) and a decrease during addition of exogenous GABA (Fig. $2 A, B$ ). It was manifest as a vertical shift in the gain functions describing drug-versus-control responses (Figs. $1 E, 2 E$ ) and was quantified by the position of the intercept on the abscissa of each linear regression. This constitutes an additive (subtractive) change in discharge rate.

The slope and intercept from linear fits to the gain functions were used to transform IPD functions, scaling down to the control function when GABA was blocked (Fig. $1 F$ ), and scaling the control functions down to GABA levels, when exogenous GABA was added (Fig. $2 F$ ). Once scaled in this manner, IPD functions recorded under control, drug, and recovery conditions appear very similar; there were no significant deviations in average scaled discharge rate ( $z$ test; $p>0.05$ ) when these linear factors were applied to the drug-affected delay functions. Without any additional normalization, it is clear that there is little difference in ITD tuning width measures once possible gain factors are accounted for. The scaled IPD functions were then normalized (Figs. 1G, 2G), only to facilitate the calculation of $50 \%$ tuning width (Figs. $1 \mathrm{H}, 2 \mathrm{H}$ ). In contrast to the nonscaled IPD functions, once the drug-induced changes in discharge rate were properly accounted for by the linear scaling procedure, there was little or no change in the $50 \%$ tuning width of functions recorded under drug conditions compared with control and recovery states.

Recovery of control discharge rates after iontophoresis of gabazine or GABA is confirmed by plotting discharge rates as a function of control discharge rates after termination of the ejection current (Figs. $1 E, 2 E ; \triangle$ ). Gain functions plotting recovery versus predrug discharge rates, predictably, lie close to unity (Figs. 1E, 2E; dotted lines).

Another important feature of the ITD functions in Figure 1 is the asymmetry around the peak response; the function on the side facing negative ITDs has a steeper slope than the function on the side facing positive ITDs. Such asymmetries, previously reported in IC responses to ITDs in noise stimuli (Kuwada et al., 1997; McAlpine et al., 2001), have been considered to be the result of selective sharpening of ITD functions around midline (i.e., close to zero) ITDs by GABAergic inhibition from the DNLL. The DNLL receives ITD-sensitive input from the medial superior olive (MSO) on the same side of the brain, and projects to both ICs, although with a bias favoring the contralateral IC. Thus, ITD-sensitive inputs from the DNLL to the IC might be expected to reflect more the opposite MSO to that providing excitatory inputs to the IC (Kuwada et al., 1997). The potential influence of such an inhibitory input was examined by measuring the slope of the ITD function. A measure of the steepness of the ITD function was obtained by measuring the slope of the function over three data points around each ITD (i.e., each ITD and its two immediate neighbors). The steepest slope on the side of the function facing negative ITDs (i.e., left of the peak response in Fig. $1 G$ ) was compared with the steepest slope on the side facing positive ITDs (i.e., right of the peak response in Fig. 1G). Blocking GABAergic inhibition had no effect on the relative steepness of the ITD function on either side of the peak response. The relatively steeper slope facing negative ITDs (ratio of 1.38:1 compared with the slope facing positive ITDs) before gabazine was applied $(\bigcirc)$ was maintained during GABA block $(\mathbf{)}$ with a ratio $1.45: 1$.

\section{Influence of GABAergic inhibition on sensitivity to ITDs in noise in IC neurons}

Responses of two IC neurons to interaurally delayed noise (NDFs) before $(\bigcirc)$, during $(\bigcirc)$, and after recovery from $(\triangle)$ iontophoresis of gabazine (top row) or GABA (bottom row) are shown in Figure 3. In each case, control NDFs showed a main peak and damped side peaks (Fig. $3 A, F$ ), consistent with previous reports (Yin et al., 1987). There was also a strong asymmetry around the main peak of the NDF, with the slope on the side facing negative ITDs being steeper than that facing positive ITDs. For the neuron in the top row, the ratio of the steepest slope on either side of the peak was 1.42:1, favoring the side facing negative ITDs. For the neuron in the bottom row, the ratio was 1.85:1. Neither the damping nor the asymmetry were altered when 

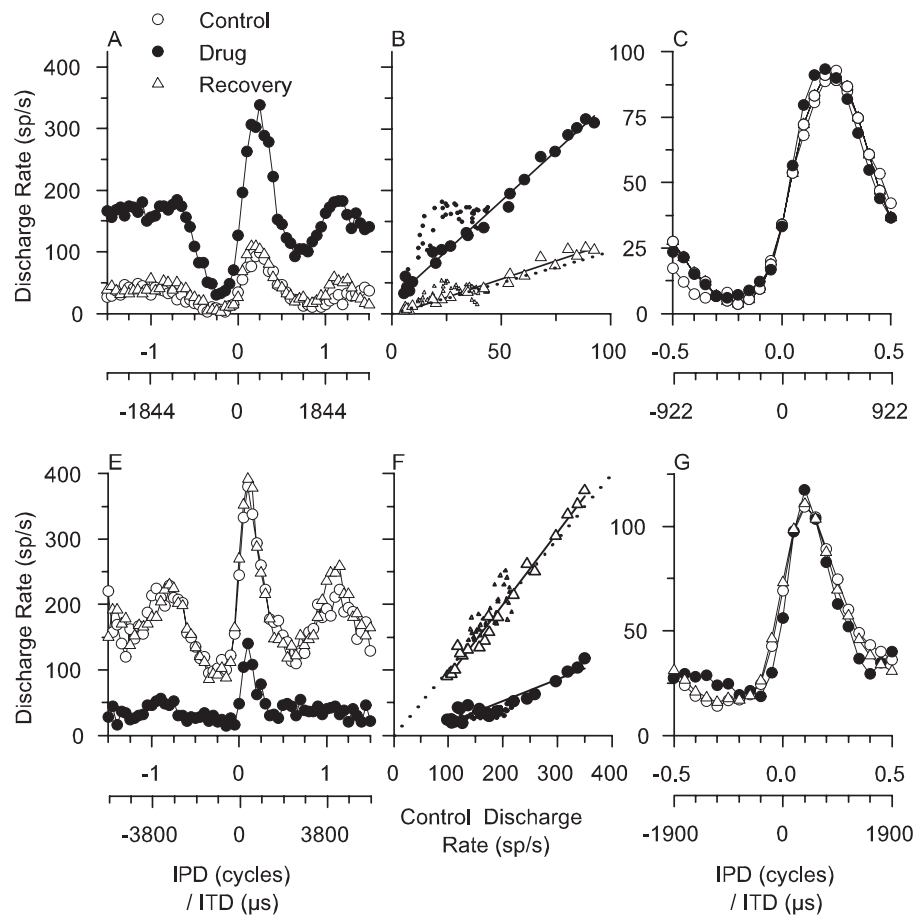

Figure 3. NDFs from IC neurons before, during, and after recovery from the effects of gabazine and GABA. Control, drug, and recovery functions are indicated by the key. The top row indicates results from application of GABA antagonist. $A$, Raw NDFs recorded over \pm 1.5 cycles IPD (with regard to characteristic frequency of the neuron). $\boldsymbol{B}$, Gain functions under drug and recovery conditions (excluding small points, from IPDs less than -0.25 cycles and more than 0.75 cycles). C, NDFs (plotted over \pm 0.5 cycles IPD) scaled down to control levels. $D$, Fifty percent tuning width (cycles of IPD) of scaled NDFs. The bottom row indicates results from iontophoresis of GABA. $\boldsymbol{E}-\boldsymbol{H}$ follow the same scheme as those described above for $\boldsymbol{A}-\boldsymbol{D}$. sp, Spike; C, control; R, recovery.

GABAergic inhibition was blocked (asymmetry ratio, 1.38:1 for the neuron in the top row of Fig. 3) or when exogenous GABA was added (1.66:1 for the neuron in the bottom row of Fig. 3). Gain functions (Fig. 3B, $F$ ) indicated multiplicative (or divisive) changes in gain during iontophoresis $(\mathbf{O})$, producing well scaled functions $(C, G)$ with little or no change in ITD tuning width $(D, H)$.

\section{GABAergic inhibition and dynamic binaural cues: responses to binaural beats}

A common stimulus used to quantify delay sensitivity in binaural neurons is the binaural beat, created using a small (usually $1 \mathrm{~Hz}$ ) difference in the frequency of tones presented to the two ears. The dynamic change in IPD that results generates the percept of sound motion. Most studies using binaural beats report similar delay sensitivity to the dynamic IPDs of binaural beats as to static ITDs (Yin and Kuwada, 1983). Figure 4, $A$ and $F$, shows responses of two IC neurons to a $3 \mathrm{~s}$ binaural beat stimulus. Iontophoresis of gabazine increased discharge rates above control levels (Fig. $4 A$, ). Responses to two complete cycles (500-2500 ms) of the beat response in Figure $4 A$ were averaged and plotted as a function of IPD in Figure $4 B$. This neuron differs from those previously in that it showed a peak response at negative, rather than positive, delays.

Blocking GABAergic inhibition scaled the discharge rate multiplicatively, with little additive shift (Fig. $4 C, \mathbf{O}$ ). Using the slope of the gain function to scale the response (Fig. $4 D$ ) revealed little change in ITD tuning $(E)$. Note also that the relative symmetry of the function around the peak response, which was opposite to that observed for neurons with peak responses at positive delays, was maintained during GABA block.
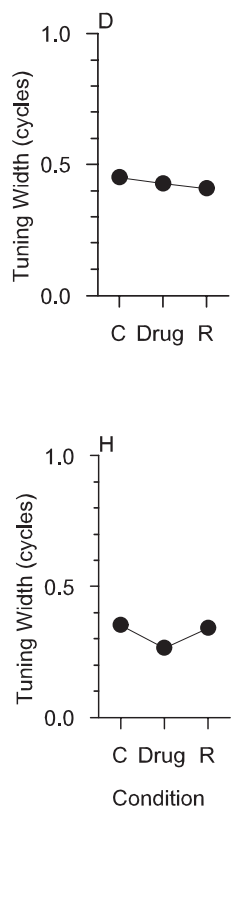

Ionotophoretic application of exogenous GABA reduced discharge rate in response to the binaural beat stimulus (Fig. $4 F, G$; ), in this case, apparently by divisive scaling $(H, \mathbf{O})$. Once the IPD functions were scaled (Fig. 4I), there was no discernible change in 50\% tuning width $(J)$. Again, the asymmetry of the function was maintained after the addition of GABA.

In both cases, excellent recovery of predrug neural responses to the binaural beat was obtained after termination of the iontophoretic current (Fig. 4, $\triangle$ ).

\section{Summary of GABAergic effects of ITD tuning in IC neurons}

Iontophoretic blockade of GABA receptors and addition of exogenous GABA had predictable effects on the discharge rates evoked during stimulation with interaurally delayed tones and noise and with binaural beats. Application of GABA receptor antagonists increased average discharge rates of TDFs $(n=14)$, NDFs $(n=4)$, and binaural beats $(n=9)$ (Fig. $5 A, D, G$, respectively), which returned to control levels on recovery from drug application. Similarly, application of GABA produced a reduction in average discharge rates of TDFs $(n=11)$, NDFs $(n=1)$, and binaural beats $(n=4)$ (Fig. 6A, $D, G$, respectively).

The mean best IPD for the population of neurons was unaffected by iontophoretic blockade of GABAergic inhibition or iontophoretic application of exogenous GABA, either to stimulation by interaurally delayed tones, interaurally delayed noise, or binaural beats (Figs. $5 B, E, H ; 6 B, E, H$; $)$ ). For TDFs, the absolute change in mean IPD compared with predrug responses was 0.029 cycles $( \pm 0.037)$, and 0.019 cycles $( \pm 0.008)$ compared with recovery functions during GABA block. Additionally, there was little influence on best IPDs to binaural beats, with an absolute change in mean best IPD of 0.014 cycles $( \pm 0.01)$ compared with predrug responses and $0.006( \pm 0.006)$ compared with recovery functions. These changes were similar to those observed during iontophoretic application of GABA itself [e.g., $0.027( \pm 0.022)$ for TDFs during GABA iontophoresis compared with predrug responses]. Because application of GABA itself should not have ITD-dependent effects, this suggests that these small changes in best IPD are mostly within the variability of repeated measurements of ITD functions. In addition, there was no effect of application of either class of drug, on the position (in terms of cycles of IPD) at which the neural response reached $50 \%$ of maximum, on either side of mean best phase (50\% border position) (Figs. $5 B, E, H ; 6 B, E, H ; \bigcirc)$. The $50 \%$ tuning width of the TDF, NDF, and binaural beat functions were similarly unaffected by application of GABA antagonist or GABA (Figs. 5C, F, I; 6C, F, I; O). Together, these observations form a compelling argument that GABAergic inhibition in the guinea pig IC does not play a role in sculpting neural responses to interaurally delayed sounds in terms of their tuning sharpness or in terms of the positioning of their receptive fields in ITD/IPD space. Additionally, large increases in discharge rate on GABA block were not accompanied by changes in the symmetry of ITD functions, which has previously been proposed to 

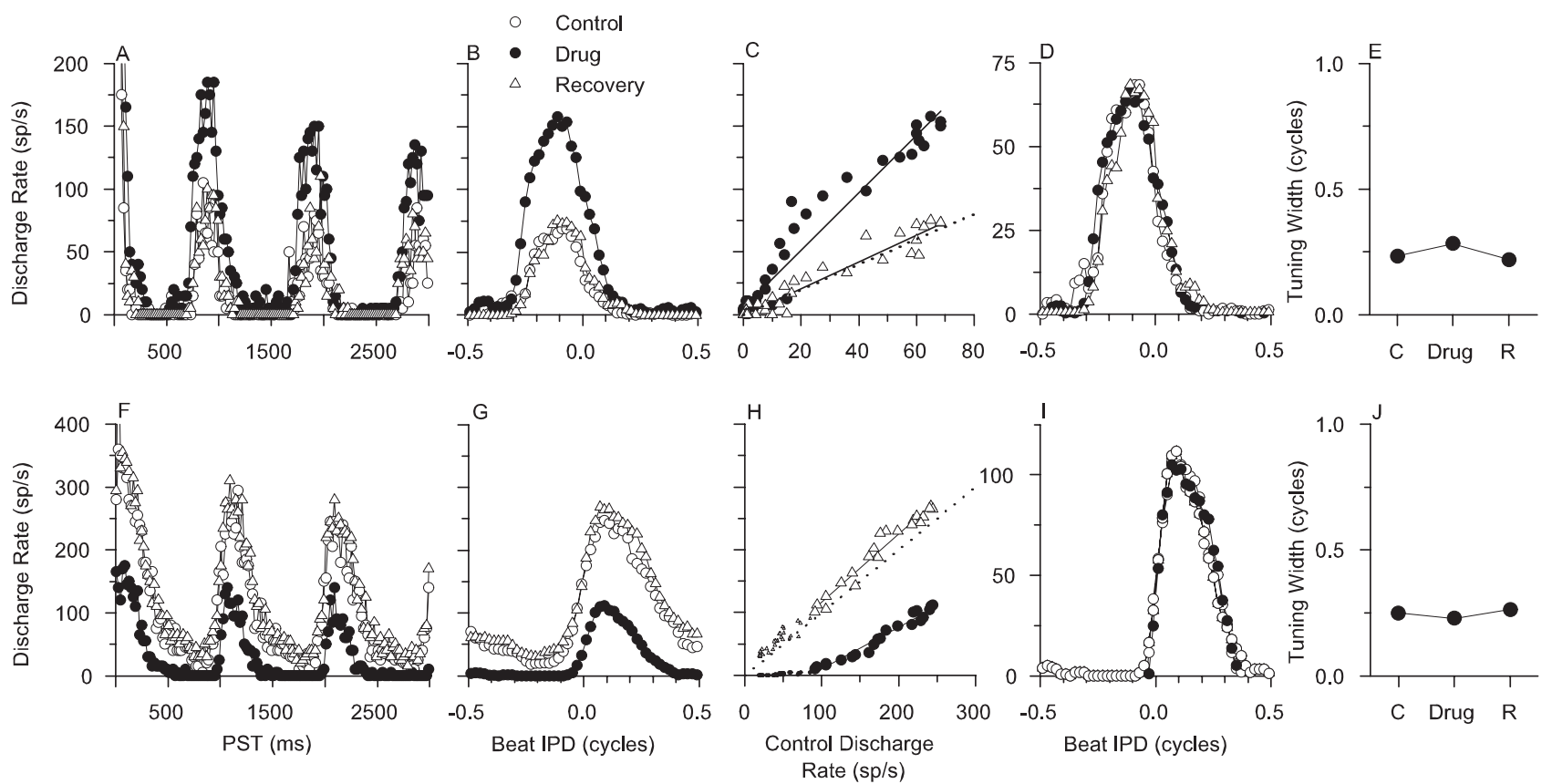

Figure 4. Binaural beat responses from IC neurons exposed to GABA and gabazine. Control, drug, and recovery functions are indicated by the key. The top row indicates results from iontophoresis of gabazine. $\boldsymbol{A}$, Raw poststimulus time (PST) histograms to three cycles of the binaural beat stimulus. $\boldsymbol{B}$, Smoothed beat period histograms, plotted relative to the IPD ( \pm 0.5 cycles) of the binaural beat stimulus. $\boldsymbol{C}$, Gain functions under drug and recovery conditions. $\boldsymbol{D}$, Beat period histograms scaled down to control levels. $\boldsymbol{E}$, Fifty percent tuning width (cycles of IPD) of scaled IPD functions. The bottom row indicates results from application of GABA. $\boldsymbol{F}$-J follow the same scheme as those described for $\boldsymbol{A}-\boldsymbol{E}$. sp, Spike; C, control; R, recovery.

reflect ITD-sensitive GABAergic inputs from the DNLL sculpting ITD functions in the IC by means of lateral (in ITD terms) inhibition.

Serial recordings in the same neuron, and effects of discharge rate adaptation/saturation

In many neurons, a series of sequential recordings were made demonstrating the effect of increasing drug concentration (i.e., increasing ejection current or increasing time after the onset of the ejection current), and in some cases, recordings were made during the exposure to both drug classes, with an intervening period of recovery. Figure 7 illustrates two such examples.

The neuron in the top row of Figure 7, $A-C$, was exposed, first to GABA, and then to bicuculline. From control conditions (Fig. 7A,, ), GABA was ejected using currents of 20, 10, and $5 \mathrm{nA}$ (Fig. 7A; see key). For the larger GABA ejection currents, there was a larger reduction in discharge rate compared with control conditions (down to $<6 \%$ of the control rate) (Fig. $7 A-C)$, which recovered to control levels after termination of the ejection current (Fig. $7 A-C$ ). Only very minor changes in $50 \%$ tuning width were noted under these conditions $(<0.05$ cycles IPD) (Fig. $7 C)$. The reduction in discharge rate was brought about primarily by divisive changes in the gain functions (Fig. $7 B$ ). The slope fell from 1.0 in control conditions (by definition) to 0.07 with $20 \mathrm{nA}$ GABA. The slope
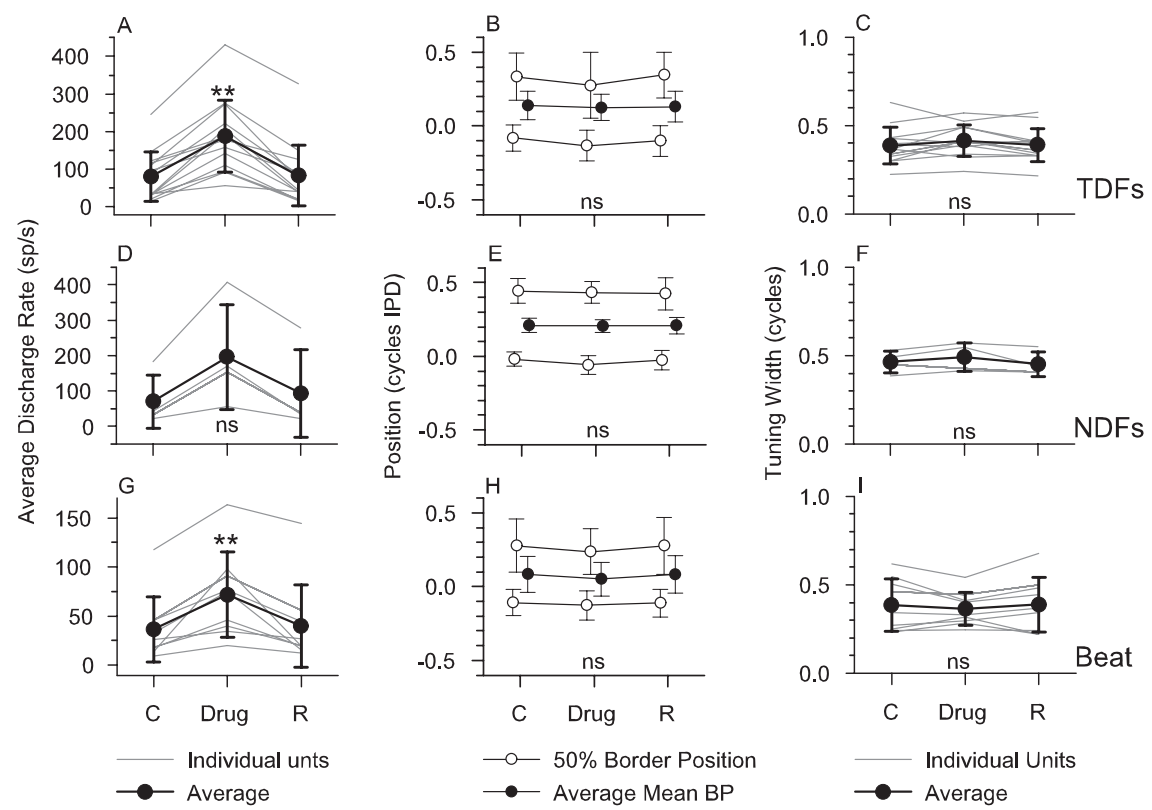

Figure 5. Summary of the effects of GABA blockade on IPD tuning. Top, middle, and bottom rows indicate data from TDFs $(\boldsymbol{A}-\boldsymbol{C}), \mathrm{NDFs}(\boldsymbol{D}-\boldsymbol{F})$, and binaural beats $(\boldsymbol{G}-\boldsymbol{I})$, respectively. Changes in average discharge rate are indicated in the left column $(\boldsymbol{A}$, $\boldsymbol{D}, \boldsymbol{G})$. Gray lines indicate data from individual neurons. Black symbols and the line indicate mean of group data ( \pm SD). Effects of drug application on IPD tuning are indicated in the middle and right columns. $\boldsymbol{B}, \boldsymbol{E}, \boldsymbol{H}$, Group-averaged mean best phase ( \pm SD) is indicated by black circles. Open circles represent group average ( $\pm S D$ ) for IPD positions at which the response crosses $50 \%$ of the maximum point for borders at IPDs above and below the mean best phase. $C, F, I$, Fifty percent tuning widths of IPD functions are plotted. Gray lines indicate data from individual neurons. Black circles and lines indicate the group average tuning width ( $\pm S D$ ). "ns" indicates no significant difference between drug and control/recovery conditions. Significant effects (ANOVA) are indicated as follows: ${ }^{*} p<0.05$ and ${ }^{* *} p<0.01$. BP, Best phase; sp, spike; C, control; R, recovery.

increased to 0.19 with $10 \mathrm{nA}$ GABA, to 0.34 with $5 \mathrm{nA} \mathrm{GABA}$, and recovered to 1.01 after the termination of the GABA ejection current. This implies that primarily multiplicative gain changes were observed in this neuron with application of GABA. 

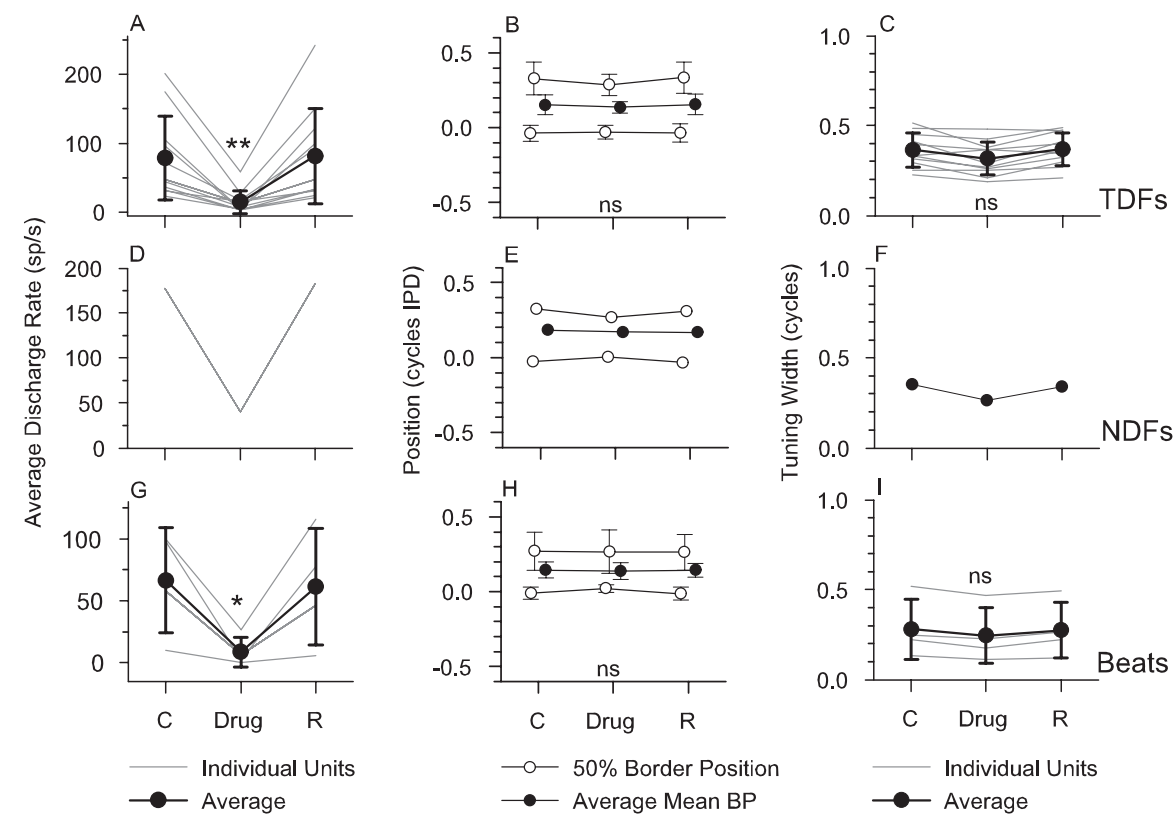

Figure 6. Summary of the effects of GABA on IPD tuning. Top, middle, and bottom rows indicate data from TDFs (A-C), NDFs $(\boldsymbol{D}-\boldsymbol{F})$, and binaural beats $(\mathbf{G}-\boldsymbol{I})$, respectively. Figure follows conventions detailed for Figure 5.
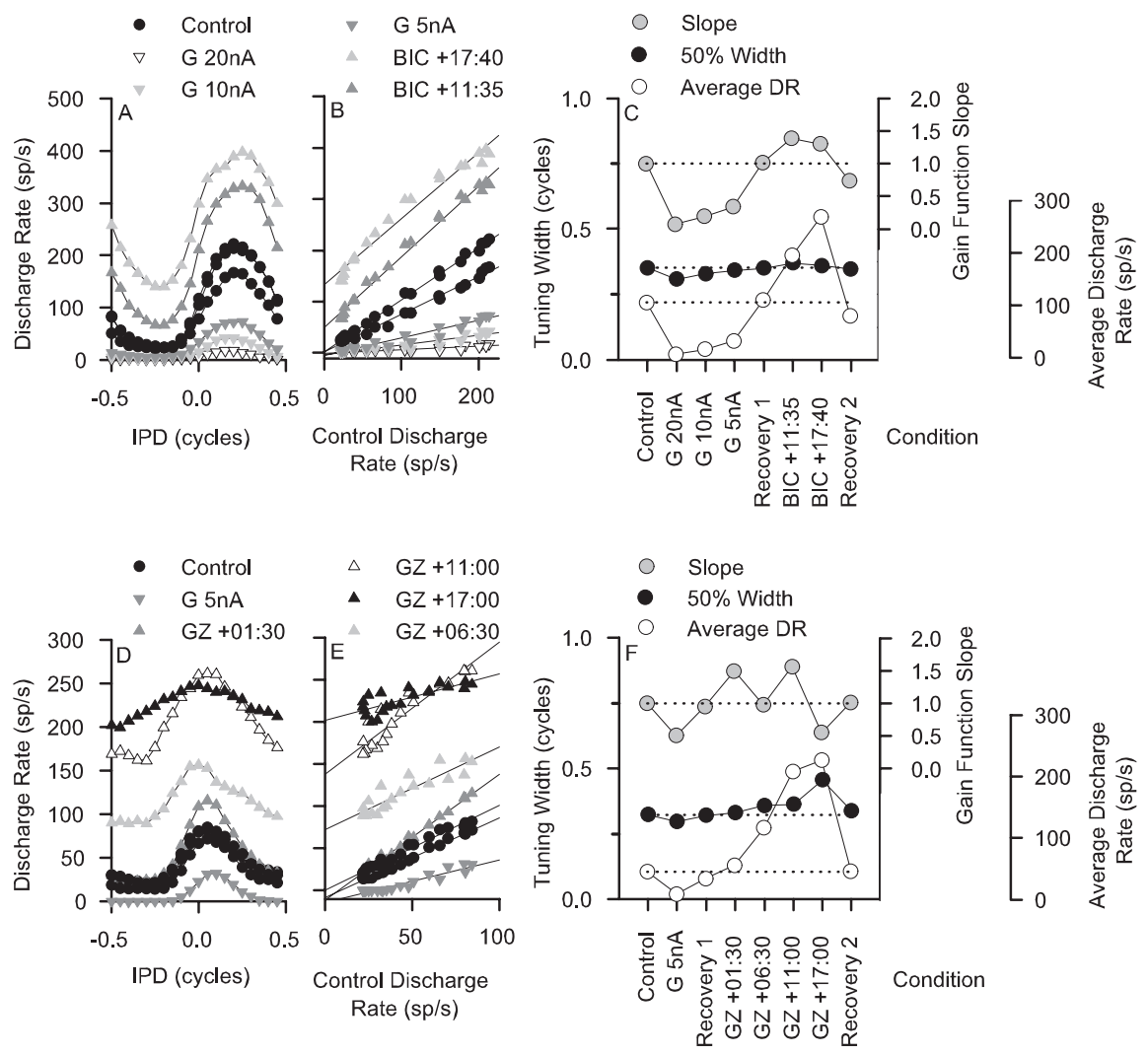

Figure 7. Serial recordings from individual neurons in response to GABA and GABA block. Folded ITD functions are shown for two neurons in $\boldsymbol{A}$ and $\boldsymbol{D}$. Filled circles indicate control and recovery responses; triangles indicate responses with iontophoresis of GABA (labeled G in key) or GABA antagonist (labeled BIC or GZ in key). In the key, iontophoresis of GABA (G) is labeled additionally with the ejection current used; iontophoresis of GABA antagonist is labeled additionally with the time after the onset of the drug ejection current. Gain functions are shown in $\boldsymbol{B}$ and $\boldsymbol{E}$ for the same neurons. $\boldsymbol{C}$ and $\boldsymbol{F}$ show composite plots illustrating changes in $50 \%$ tuning width, average discharge rate, and gain function slope (indicated in key) with changing drug/recovery condition, from control, through exposure to GABA, recovery from GABA effects (recovery 1), GABA blockade, and finally to recovery from all iontophoresed drugs (recovery 2). Dotted lines indicate control value for each parameter for comparison at each drug condition. sp, Spike; DR, discharge rate.
When $25 \mathrm{nA}$ of bicuculline ejection current was applied to the same neuron, discharge rate increased to $>250 \%$ of control levels after $17 \mathrm{~min}$ and $40 \mathrm{~s}$ of current application (Fig. $7 A-C$; see key). There was a modest increase in the slope of the gain function (Fig. $7 B, C$ ) from 1.01 to 1.38 over the same time period, indicating some multiplicative gain, and a substantial increase in the baseline, ITD-insensitive, discharge rate, indicating some additive gain. Despite these dramatic discharge rate effects, there was negligible effect on the $50 \%$ tuning width of the functions $(<0.02$ cycles increase) (Fig. 7C). When the bicuculline ejection current was terminated, these parameters returned to levels close to the initial control recordings.

A second example of serial recordings is shown in the bottom row of Figure 7, D-F. From control levels, $5 \mathrm{nA}$ GABA ejection current was applied and there was a resulting fall in discharge rate (Fig. $7 D-F$ ). Concomitantly, the slope of the gain function was reduced from 1.0 to 0.50 , with little offset shift, indicating a primarily divisive gain change. However, there was, again, negligible effect on $50 \%$ tuning width of the scaled ITD functions (from 0.32 to 0.30 cycles). On termination of the GABA current, discharge rate recovered, the slope of the gain function returned close to unity (0.95), and the 50\% width returned to 0.32 cycles. When a gabazine ejection current was applied, to block inhibition, there was a clear increase in discharge rate, with the largest rates recorded $17 \mathrm{~min}$ after the onset of the current. For this series of recordings, sustained blockade of GABAergic inhibition pushed the neurons discharge rate into saturation. After 11 min of gabazine ejection current, the neuron maintained a response that was well modulated with ITD (Fig. $7 D, \triangle$ ). However, as the average discharge rate increased further with time, the response became less well modulated with ITD (Fig. $7 D, \mathbf{\Delta})$. There was also a tendency for peak responses to fall slightly below those of the ITD function recorded at $11 \mathrm{~min}$ into the ejection current (with a lower average discharge rate). We interpret these observations as the effects of saturation on the input-versus-output function of the neuron [firing rate $(f)$-input current $(I)$ curve] (see Discussion) and possibly spike frequency adaptation. The randomized presentation of different ITDs coupled with the high spike rate at all ITDs means that the neuron is likely forced into an adapted state, even at less favorable ITDs. Because recovery from adaptation in ITDsensitive IC neurons is a slower process 
than the adaptation itself (Ingham and McAlpine, 2004), the relatively fast repetition rate of ITD presentation (250 ms duty cycle) means that the neuron was not able to recover from the adaptation induced by the high spike rates, resulting in spike rates at favorable ITDs that could be lower than those recorded for the same ITDs in functions in which the average discharge rate was lower. Strictly, we cannot differentiate between saturation and adaptation effects, but the higher baseline rate and lower peak rate had a significant effect on the gain function describing this particular condition for the neuron. As average discharge rate increased, the slope of the gain function was seen to increase from 0.95 to 1.56 (after $11 \mathrm{~min}$ ) with accompanying increases in function offset (Fig. 7E), indicating a combined additive and multiplicative gain control. With an additional increase in average discharge rate (at 17 $\mathrm{min}$ ), the gain function flattened, showing a slope of only 0.55 and a large offset. This effect is consistent with the influence of saturation and/or spike frequency adaptation described above. It should be noted that the $50 \%$ tuning width is principally unaffected by the addition of gabazine, increasing from 0.32 cycles (recovery 1 ) to 0.36 cycles (after $11 \mathrm{~min}$ of gabazine ejection). Only when the ITD modulation of discharge rate breaks down, and thus the gain function scaling of the function performs less well, is there any degree of change in $50 \%$ tuning width (increasing to 0.46 cycles after $17 \mathrm{~min}$ ). However, this tuning width measure returns to 0.34 cycles when the neuron was fully recovered from the effects of gabazine. Similarly, after the termination of the gabazine current, the gain function slope returned to control values as did discharge rate (Fig. $7 F$ ).

These serial recordings of the effects noted provide additional evidence that GABAergic inhibition in the IC serves to regulate the output of the IC neurons with no apparent ITD-specific effects on the sharpness of ITD tuning in these neurons. It should be noted that any recordings obtained indicating strong evidence of saturation/adaptation effects were not included in the summary data shown in Figure 5.

\section{GABAergic inhibition and ITD discrimination}

Although GABAergic inhibition does not sharpen ITD functions by lateral inhibition-it has no effect on preferred interaural delays or $50 \%$ tuning widths - it does, nevertheless, modulate ITD discrimination thresholds, as would be expected from a mechanism that adjusted neural discharge rates
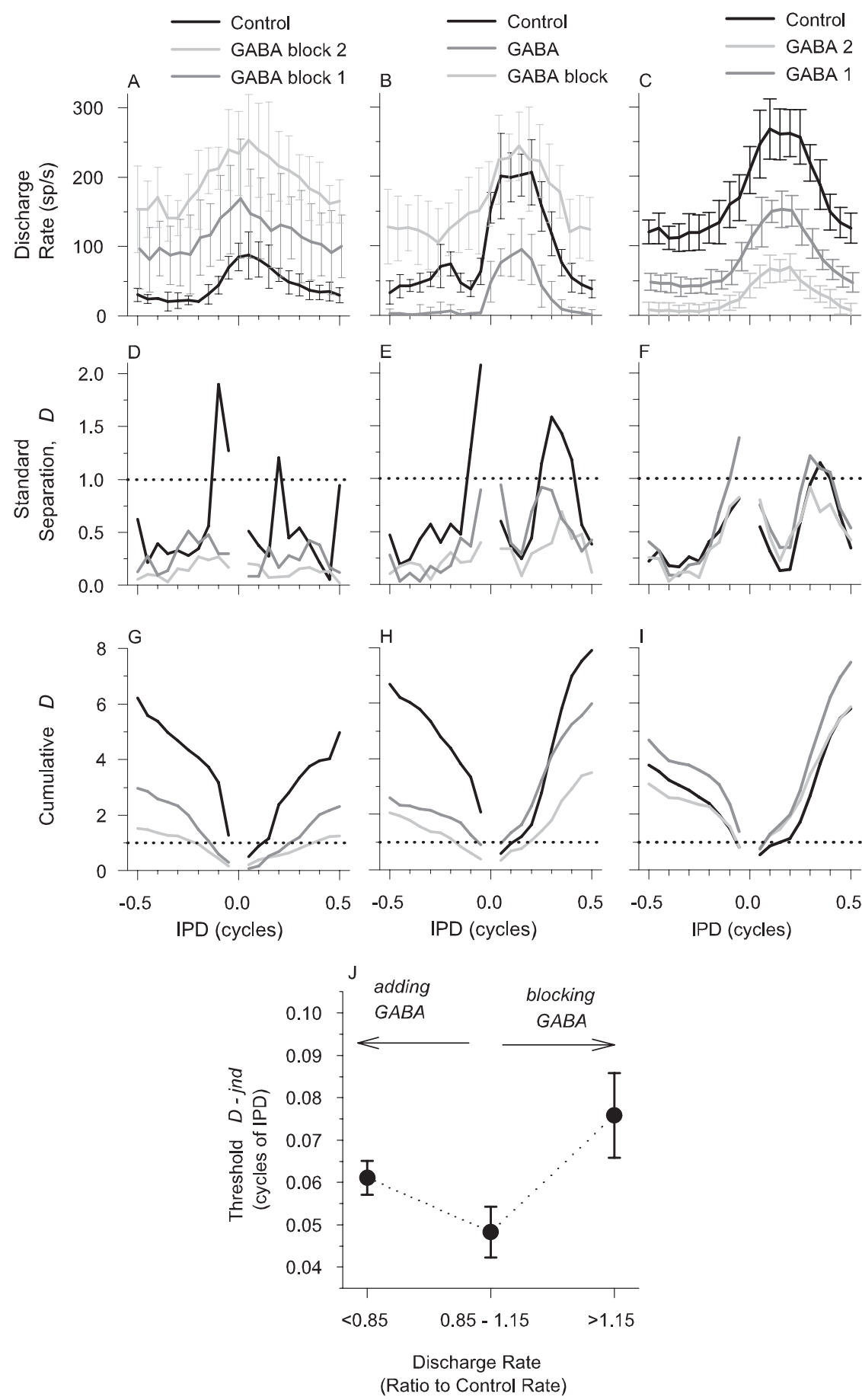

Figure 8. ITD discrimination by single neurons, the responses of which were modified by blocking, or adding exogenous, GABA inhibition. $A-C$, Folded ITD functions for three neurons, indicating average discharge rate $( \pm S D)$. For the neuron in the left column $(\boldsymbol{A}, \boldsymbol{D}, \boldsymbol{G})$, data are plotted for two levels of GABA blockade (gray lines) as well as a control (black line). For the neuron in the middle column $(\boldsymbol{B}, \boldsymbol{E}, \boldsymbol{H})$, data are plotted for responses during iontophoresis of gabazine (light gray lines) and during iontophoresis of GABA (dark gray lines). For the neuron in the right column $(C, F, I)$, data are plotted for two different GABA ejection currents (gray lines) and control (black line). $\boldsymbol{D}-\boldsymbol{F}$, The standard separation, $D$ (see Materials and Methods), for each ITD away from zero cycles is plotted for the three neurons. $\mathbf{G}-\boldsymbol{I}$, Cumulative $D$ (for functions in $\boldsymbol{D}-\boldsymbol{F}$ ) is plotted as ITD increases from zero cycles. Dotted lines indicate a value of 1.0 , above which ITD becomes discriminable from zero. J, Averaged threshold $D$ — the jnd — calculated from TDFs plotted as a function of discharge rate ratio (values $<0.85$ indicating discharge rates during iontophoresis of GABA; values $>1.15$ indicating discharge rates during GABA blockade). Error bars represent SEM.

per se. Figure $8, A-C$, shows responses of three IC neurons to interaurally delayed tones. Blocking or adding GABAergic inhibition modulated discharge rates and produced multiplicative and/or additive changes in neural gain (as described above) (gain 

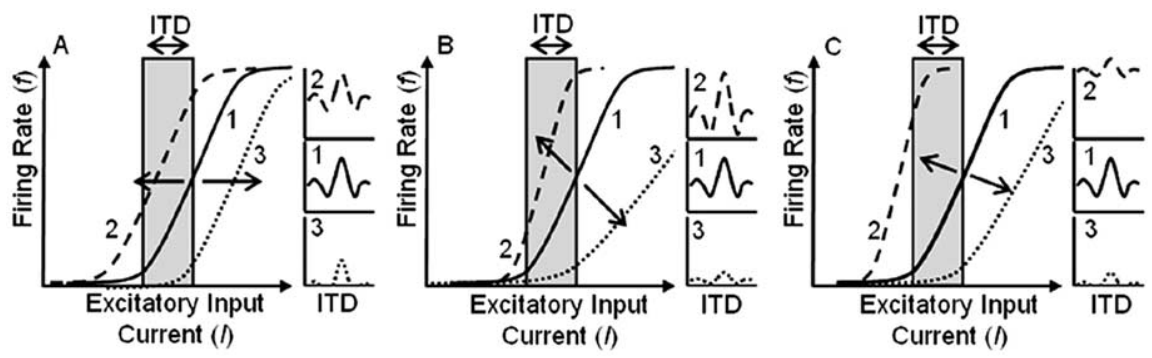

Figure 9. Gain control models. $\boldsymbol{A}-\boldsymbol{C}$, Main panel indicates input-output or $f$-l curves, under control conditions $(1$, solid line), conditions of reduced GABA (2, dashed line), and conditions of additional GABA (3, dotted line). The gray area indicates the range of input current produced by an ITD-modulated stimulus and the corresponding range of neural output. Small panels on the right indicate ITD functions that might be produced under the same conditions.

functions not shown). These gain changes were accompanied by altered ITD discrimination performance. For each neuron, a measure of discrimination-the standard separation or $D$ (see Materials and Methods) — was calculated over ITDs equivalent to \pm 0.5 cycles of IPD around zero delay, corresponding to the auditory midline. Discrimination at each delay was calculated between neighboring values in either direction away from zero delay. Values of $D<1.0$ (Fig. $8 D-F$, horizontal lines) are considered below discrimination threshold. Consistent with recent reports in the guinea pig (Shackleton et al., 2003), sounds leading at the ipsilateral (to the IC) ear are most discriminable at the neural level. Figure $8, D-F$, indicates that, under control conditions (black lines), a shift of 0.05 cycles from zero toward negative (ipsilateral leading) delays were more detectable (i.e., higher $D$ value) than a shift of 0.05 cycles toward contralateral delays.

For the neuron shown in the left column of Figure 8, blocking GABAergic inhibition (gray lines) reduced discrimination across the range of delays as discharge rate increased, particularly when, with sustained gabazine current, discharge rates started to saturate at favorable delays (light gray line). This is reflected in the lower $D$ values for these conditions, plotted in Figure $8 D$. The same effect was noted for the neuron plotted in the middle column of Figure 8. Blocking inhibition (light gray line) increased discharge rate (Fig. $8 B$ ) and reduced $D$ values (i.e., discrimination) across all ITDs compared with the control data (Fig. $8 E$, black line).

Discrimination was usually reduced with iontophoresis of exogenous GABA (Fig. 8, middle column, dark gray lines), presumably as spike count decreased and the peak-to-trough modulation depth of the delay function was reduced. Figure $8 E$ (dark gray lines) indicates that $D$ values were reduced at all ITDs compared with control values (black lines).

It was not always the case that control levels of GABA provided for best discrimination. The neuron in the right column in Figure 8 , for example, showed a large ITD-insensitive component to its control response (black line), which was substantially reduced during iontophoresis of GABA (gray line). This increased the proportion of the response that was modulated with interaural delay, and improved the discrimination performance of the neuron (Figure $8 F$, gray line). However, with an additional increase in the GABA current (light gray line), discharge rate continued to fall, and discrimination was reduced as response modulation, in terms of absolute discharge rate, decreased.

Recent data suggest that slopes of interaural delay functions, rather than their broad peaks, are a critical factor in sound localization over the frequency range at which sensitivity to interaural delays is observed in mammals (Shackleton et al., 2003). These slopes are often positioned across the physiological range of ITDs $( \pm 180 \mu$ s in guinea pigs) around zero delay, with the result that modulating the interaural delay of a sound source, or head movement with respect to a fixed sound source, modulates the discharge rate up and down the slope of delay function. For each neuron, the "just-noticeable difference" (jnd)-the minimum detectable change in interaural delay from zero-was obtained by calculating the cumulative $D$ either side of zero delay. The $D$ value previously calculated between neighboring ITDs was summed for consecutive ITDs in either direction away from zero delay. The interaural delay at which $D$ crossed the threshold value of 1.0 was considered the threshold interaural delay-the smallest change in ITD from zero that could be detected. This analysis is shown in the bottom row for each of the neurons in Figure 8. For the neuron in the left column of Figure 8 , the minimum detectable change in ITD from zero (Fig. 8G), the threshold jnd from zero, corresponded to 0.039 cycles, equivalent to a minimum detectable interaural time difference of $78 \mu$ s at $500 \mathrm{~Hz}$, the tone frequency used to obtain the delay function. As discharge rate increased with increasing GABA block, the minimum detectable change in IPD increased to 0.22 cycles ( $435 \mu$ s; dark gray line) and, finally, to 0.25 cycles $(505$ $\mu \mathrm{s}$; light gray line) when the interaural delay function was close to saturation. For the neuron in the middle column of Figure 8, the jnd increased from 0.024 cycles (in control conditions) to 0.064 cycles when additional GABA was applied, or to 0.178 cycles when GABA was blocked (Fig. $8 H$ ). For the neuron in the right column of Figure 8, the minimum detectable change in ITD was obtained with the intermediate discharge rate evoked during GABA block (Fig. 8I).

The tendency for thresholds to be lowest for control levels of GABAergic inhibition is confirmed in Figure $8 \mathrm{~J}$, which shows the effect of blocking or adding exogenous GABA on minimum discriminable interaural delay in response to interaurally delayed pure tones for 15 neurons. Mean threshold ITD was elevated as discharge rate increased ( $>15 \%$ increase cf. control) with iontophoresis of gabazine $(0.076 \pm 0.044$ cycles of IPD) or as discharge rate decreased $(>15 \%$ decrease $\mathrm{cf}$. control) with iontophoresis of GABA $(0.061 \pm 0.029$ cycles of IPD) compared with the mean threshold for control discharge rates $(0.048 \pm 0.020$ cycles of IPD). ANOVA revealed these differences to be significant $(F=$ 4.55; $p<0.02)$.

\section{Discussion}

The first finding of this study is that GABAergic inhibition regulates the output gain of IC neurons sensitive to ITDs, such that discharge rates undergo multiplicative/divisive scaling, additive/ subtractive offsets, or both. Iontophoresis of GABA antagonists increased spike rates by variable amounts, often proportional to control rates, at each ITD. Iontophoresis of GABA had the opposite effect to iontophoresis of gabazine. The relationship between discharge rates evoked during drug and control conditions were well described by linear regression fits, the parameters of which were used to scale discharge rates to equivalent levels.

Figure 9 illustrates how neural gain might be controlled by GABAergic inhibition. Solid lines (1) in the main panels of Figure 9, $A-C$, indicate hypothetical underlying "input-output" relationships, in which as $I$ increases, neural output $(f)$ increases in a 
sigmoidal manner. This form of function is often referred to as an $f-I$ curve. Under control conditions, ITD-tuned input current modulates firing rate over a specific input range (gray area) to produce an output, in this case, an ITD function, which is shown in the middle of the small panels (1) on the right of each main panel.

Multiplicative gain control scales firing rate such that the slope of the $f-I$ curve is increased (Fig. $9 A$, dashed line, 2) or decreased (divisive gain) (Fig. 9A, dotted line, 3). Under these circumstances, the slope of the $f-I$ curve is altered, modifying the range of firing rates evoked by the ITD-tuned input current. This produces an up-scaled or down-scaled ITD function (small panels, 2 and 3, respectively), in which each ITD-evoked discharge rate is multiplied by a common factor. Thus, ITD functions are scaled versions of each other, "stretching" as GABA inhibition is blocked and "compressing" with the addition of exogenous GABA. Such discharge rate-dependent gain could involve ITDdependent inhibitory inputs in which the strength of inhibition is proportional to that of the excitatory input.

Additive gain (Fig. 9B) shifts the $f-I$ curve from its original position (curve 1) to the left or the right, adding (curve 2) or subtracting (curve 3 ) a constant number of spikes. Thus, additive gain applies equally to every ITD. A constant (e.g., ITDinsensitive) inhibition could achieve this.

In extremis, elevating the discharge rate by blocking GABAergic inhibition could push responses into the saturated portion of the $f-I$ curve (Fig. 9C, curve 2). This effect, which can arise through either multiplicative or additive gain, would result in ITD functions being compressed at higher rates, and less well modulated with ITD. Adding exogenous GABA could also reduce the modulation depth, creating an "iceberg effect" such that only the peaks of ITD functions are observed in the extracellularly recorded response (Fig. 9C, curve 3 ). Because exogenous GABA is ITD independent, it might be presumed to have an ITDindependent effect on discharge rate, although this is not necessarily the case (see below).

\section{GABAergic inhibition and sensitivity to interaural time differences}

The second finding of this study is that GABAergic inhibition does not influence the sensitivity of IC neurons to ITDs, at least in terms in which such sensitivity is normally considered. Once changes in neural gain are accounted for, neural tuning for preferred ITD and tuning sharpness were unaffected by blocking GABAergic inhibition or by adding exogenous GABA. This is contrary to previous reports. Using a simple normalization procedure (to the peak discharge rate) of their ITD functions, Fujita and Konishi (1991) (in the barn owl) and Sterbing et al. (2005) (in the rabbit) concluded that GABAergic inhibition serves to sharpen delay tuning in IC neurons. However, such a normalization process is usually reported without mathematical justification and fails to take into account the influence of neural gain. With the assumption that GABA controls multiplicative and additive gain, changes in neural responses to static ITDs during GABA block or addition of exogenous GABA can be accounted for by scaling and/or offsetting neural responses by the appropriate factors. Reanalysis of previously published ITD functions (Fujita and Konishi, 1991) using our scaling procedure demonstrates that delay functions in barn owl IC scale in the same way as those seen in the current study, and that no changes in static ITD tuning are observed. Our data do not preclude the possibility that ITD functions may be sharper at successive stations in the ascending auditory pathway (suggested by Fitzpatrick et al., 1997), but it does not support a role for GABA in the sharpening process at the level of the IC.

\section{Potential sources of GABAergic inhibition to the IC}

Two potential circuits that could provide for ITD-sensitive inhibition are via the DNLL and via local interneurons in the IC itself. The DNLL receives input from the MSO on the same side of the brain and projects its primarily GABAergic inhibitory input bilaterally to the IC, although favoring the IC on the opposite side of the brain. Several studies have demonstrated DNLL neurons to be ITD sensitive (Aitkin et al., 1970; Brugge et al., 1970), and it is often assumed that DNLL neurons influence IC neurons in a delay-sensitive manner. Our data potentially support this contention but indicate that any delay-sensitive GABAergic input to IC neurons operates in a different manner to that previously imagined, in that it requires precise matching of the delaysensitive inhibitory and excitatory inputs to produce the multiplicative gain effects we observe. This projection has been proposed as the candidate nucleus for sculpting responses of ITD-sensitive neurons, possibly providing them with their "sawtooth" appearance (Kuwada et al., 1997), as well as for rendering IC neurons sensitive to the context in which IPD cues are presented (Spitzer and Semple, 1998). However, it was notable that no change in the shape of ITD functions occurred during GABA block; the relative symmetry around the peak responses of delay functions and binaural beats was maintained. Such asymmetries have been suggested previously to arise from the influence of a mismatch between the ITD sensitivity of the excitatory and inhibitory ITD inputs to IC neurons. However, our data demonstrate convincingly that GABAergic inhibition does not have the expected properties and appears remarkably well matched in input strength to the excitatory input across the range of excitatory input values.

An alternate and plausible explanation, given the requirement of precisely matched excitatory and inhibitory inputs, is that GABAergic inhibitory circuits, acting in feedforward or local (to the IC) feedback loops. This would provide a mechanism of gain control local to the IC that matches excitatory input levels to the range of discharge rates at which discrimination performance is best. Similarly, acoustic parameters other than binaural cues are likely to be subject to the same forms of gain control in the IC; ITD-sensitive neurons in the IC are also sensitive to changes in sound frequency and intensity. Local circuitry in the IC could modulate the efficacy of GABAergic inhibition for specific sound sources, enhancing detection and recognition of auditory objects in a complex acoustic environment.

\section{Mechanisms of gain control in the IC}

A potential candidate mechanism to control neural gain is shunting inhibition, mediated by a GABA-controlled $\mathrm{Cl}^{-}$conductance. Shunting inhibition has long been considered a potential mechanism for gain control, although initial in vitro studies appeared to demonstrate that shunting inhibition produced only subtractive gain effects, and could not account for divisive gain (Brickley et al., 1996; Holt and Koch, 1997; Chance et al., 2002). Recent in vitro studies indicate that, when input trains mimic the noisy synaptic inputs known to exist in vivo, shunting inhibition produces divisive gain (Mitchell and Silver, 2003). In the current study, the divisive gain change observed on iontophoresis of GABA is particularly relevant, because it represents a gain change that is dependent on the discharge rate, and by extension the underlying voltage-current relationship of the neuron. Given the ITD-independent nature of exogenous GABA, this is consistent 
with a cellular mechanism of gain control, rather than the involvement of feedback/feedforward circuits. Such loops cannot be excluded, however, especially given the nature of the experimental procedure, which involved blocking all potential GABAergic inputs. It is possible that interneurons themselves are influenced by exogenous GABA, and their proximity, or otherwise, to the recorded neuron is unknown, as indeed is their existence. Exogenous GABA might also reduce the excitatory influence of the recorded neuron on any inhibitory interneurons acting in a feedback loop circuit, reducing their efficacy, and resulting in a compensatory increase in neural gain. However, in the absence of detailed knowledge concerning the influence of local inhibitory circuits in the IC, and the apparent lack of inputs mismatched in their ITD sensitivity relative to that of excitatory inputs, the most parsimonious explanation is one of shunting inhibition, which does not require extensive neural circuitry, relying instead on processes restricted to the neuron itself. Recent studies suggest that IC neurons in the barn owl multiply inputs sensitive to interaural time and interaural intensity differences (Pena and Konishi, 2001), and that inhibition is likely involved in modifying these responses (Pena and Konishi, 2002). The current data are not inconsistent with this hypothesis but also suggest a broader role for neural multiplication in controlling neural gain.

\section{References}

Adams JC (1979) Ascending projections to the inferior colliculus. J Comp Neurol 183:519-538.

Adams JC (1980) Crossed and descending projections to the inferior colliculus. Neurosci Lett 19:1-5.

Adams JC, Mugnaini E (1984) Dorsal nucleus of the lateral lemniscus: a nucleus of GABAergic projection neurons. Brain Res Bull 13:585-590.

Aitkin LM, Anderson DJ, Brugge JF (1970) Tonotopic organization and discharge characteristics of single neurons in nuclei of the lateral lemniscus of the cat. J Neurophysiol 33:421-440.

Andersen RA, Roth GL, Aitkin LM, Merzenich MM (1980) The efferent projections of the central nucleus and the pericentral nucleus of the inferior colliculus in the cat. J Comp Neurol 194:649-662.

Beyerl BD (1978) Afferent projections to the central nucleus of the inferior colliculus in the rat. Brain Res 145:209-223.

Brickley SG, Cull-Candy SG, Farrant M (1996) Development of a tonic form of synaptic inhibition in rat cerebellar granule cells resulting from persistent activation of $\mathrm{GABA}_{\mathrm{A}}$ receptors. J Physiol (Lond) 497:753-759.

Brugge JF, Anderson DJ, Aitkin LM (1970) Responses of neurons in the dorsal nucleus of the lateral lemniscus of cat to binaural tonal stimulation. J Neurophysiol 33:441-458.

Brunso-Bechtold JK, Thompson GC, Masterton RB (1981) HRP study of the organization of auditory afferents ascending to central nucleus of inferior colliculus in cat. J Comp Neurol 197:705-722.

Burger RM, Pollak GD (1998) Analysis of the role of inhibition in shaping responses to sinusoidally amplitude-modulated signals in the inferior colliculus. J Neurophysiol 80:1686-1701.

Burger RM, Pollak GD (2001) Reversible inactivation of the dorsal nucleus of the lateral lemniscus reveals its role in the processing of multiple sound sources in the inferior colliculus of bats. J Neurosci 21:4830-4843.

Caspary DM, Milbrandt JC, Helfert RH (1995) Central auditory aging: GABA changes in the inferior colliculus. Exp Gerontol 30:349-360.

Casseday JH, Ehrlich D, Covey E (2000) Neural measurement of sound duration: control by excitatory-inhibitory interactions in the inferior colliculus. J Neurophysiol 84:1475-1487.

Chance FS, Abbott LF, Reyes AD (2002) Gain modulation from background synaptic input. Neuron 35:773-782.

Fitzpatrick DC, Batra R, Stanford TR, Kuwada S (1997) A neuronal population code for sound localization. Nature 388:871-874.

Friauf E, Kandler K (1990) Auditory projections to the inferior colliculus of the rat are present by birth. Neurosci Lett 120:58-61.

Fujita I, Konishi M (1991) The role of GABAergic inhibition in processing of interaural time difference in the owl's auditory system. J Neurosci 11:722-739.
Goldberg JM, Brown PB (1969) Response of binaural neurons of dog superior olivary complex to dichotic tonal stimuli: some physiological mechanisms of sound localization. J Neurophysiol 32:613-636.

Gonzalez-Hernandez T, Mantolan-Sarmiento B, Gonzalez-Gonzalez B, Perez-Gonzalez H (1996) Sources of GABAergic input to the inferior colliculus of the rat. J Comp Neurol 372:309-326.

Green DM, Swets JA (1966) Signal detection theory and psychophysics. New York: Wiley.

Holt GR, Koch C (1997) Shunting inhibition does not have a divisive effect on firing rates. Neural Comput 9:1001-1013.

Ingham NJ, McAlpine D (2004) Spike-frequency adaptation in the inferior colliculus. J Neurophysiol 91:632-645.

Irvine DRF (1992) Physiology of auditory brainstem pathways. In: Springer handbook of auditory research, $\mathrm{Vol} 2$, The mammalian auditory pathway: neurophysiology (Fay RR, Popper AA, eds), pp 153-231. New York: Springer.

Kuwada S, Batra R, Fitzpatrick DC (1997) Neural processing of binaural temporal cues. In: Binaural and spatial hearing in real and virtual environments (Gilkey RH, Anderson TR, eds), pp 399-425. Maywah, NJ: Lawrence Erlbaum Associates.

LeBeau FE, Rees A, Malmierca MS (1996) Contribution of GABA- and glycine-mediated inhibition to the monaural temporal response properties of neurons in the inferior colliculus. J Neurophysiol 75:902-919.

LeBeau FE, Malmierca MS, Rees A (2001) Iontophoresis in vivo demonstrates a key role for $\mathrm{GABA}_{\mathrm{A}}$ and glycinergic inhibition in shaping frequency response areas in the inferior colliculus of guinea pig. J Neurosci 21:7303-7312.

McAlpine D, Palmer AR (2002) Blocking GABAergic inhibition increases sensitivity to sound motion cues in the inferior colliculus. J Neurosci 22:1443-1453.

McAlpine D, Jiang D, Shackleton TM, Palmer AR (2000) Responses of neurons in the inferior colliculus to dynamic interaural phase cues: evidence for a mechanism of binaural adaptation. J Neurophysiol 83:1356-1365.

McAlpine D, Jiang D, Palmer AR (2001) A neural code for low-frequency sound localization in mammals. Nat Neurosci 4:396-401.

Mitchell SJ, Silver RA (2003) Shunting inhibition modulates neuronal gain during synaptic excitation. Neuron 38:433-445.

Ohzawa I, Sclar G, Freeman RD (1982) Contrast gain control in the cat visual cortex. Nature 298:266-268

Ohzawa I, Sclar G, Freeman RD (1985) Contrast gain control in the cat's visual system. J Neurophysiol 54:651-667.

Oliver DL (1987) Projections to the inferior colliculus from the anteroventral cochlear nucleus in the cat: possible substrates for binaural interaction. J Comp Neurol 264:24-46.

Oliver DL, Beckius GE (1992) Fine structure of GABA-labeled axonal endings in the inferior colliculus of the cat: immunocytochemistry on deplasticized ultrathin sections. Neuroscience 46:455-463.

Oliver DL, Winer JA, Beckius GE, Saint Marie RL (1994) Morphology of GABAergic neurons in the inferior colliculus of the cat. J Comp Neurol 340:27-42.

Oliver DL, Beckius GE, Shneiderman A (1995) Axonal projections from the lateral and medial superior olive to the inferior colliculus of the cat: a study using electron microscopic autoradiography. J Comp Neurol 360:17-32.

Oliver DL, Beckius GE, Bishop DC, Loftus WC, Batra R (2003) Topography of interaural temporal disparity coding in projections of medial superior olive to inferior colliculus. J Neurosci 23:7438-7449.

Pena JL, Konishi M (2001) Auditory spatial receptive fields created by multiplication. Science 292:249-252.

Pena JL, Konishi M (2002) From postsynaptic potentials to spikes in the genesis of auditory spatial receptive fields. J Neurosci 22:5652-5658.

Pollak GD, Park TJ (1993) The effects of GABAergic inhibition on monaural response properties of neurons in the mustache bat's inferior colliculus. Hear Res 65:99-117.

Sanes DH, Malone BJ, Semple MN (1998) Role of synaptic inhibition in processing of dynamic binaural level stimuli. J Neurosci 18:794-803.

Shackleton TM, Skottun BC, Arnott RH, Palmer AR (2003) Interaural time difference discrimination thresholds for single neurons in the inferior colliculus of guinea pigs. J Neurosci 15:716-724.

Spitzer MW, Semple MN (1991) Interaural phase coding in auditory midbrain: influence of dynamic stimulus features. Science 254:721-724.

Spitzer MW, Semple MN (1995) Neurons sensitive to interaural phase dis- 
parity in gerbil superior olive: diverse monaural and temporal response properties. J Neurophysiol 73:1668-1690.

Spitzer MW, Semple MN (1998) Transformation of binaural response properties in the ascending auditory pathway: influence of time-varying interaural phase disparity. J Neurophysiol 80:3062-3076.

Sterbing SJ, D'Angelo WR, Ostapoff EM, Kuwada S (2005) The role of GABA-ergic inhibition in the coding of interaural time differences of low-frequency sounds in the inferior colliculus. J Neurophysiol 93:3390-3400.

Takahashi TT, Konishi M (1988) Projections of the cochlear nuclei and nucleus laminaris to the inferior colliculus of the barn owl. J Comp Neurol 274:190-211.
Winer JA, Chernock ML, Larue DT, Cheung SW (2002) Descending projections to the inferior colliculus from the posterior thalamus and the auditory cortex in rat, cat, and monkey. Hear Res 168:181-195.

Yin TC, Kuwada S (1983) Binaural interaction in low-frequency neurons in inferior colliculus of the cat. II. Effects of changing rate and direction of interaural phase. J Neurophysiol 50:1000-1019.

Yin TC, Chan JC, Carney LH (1987) Effects of interaural time delays of noise stimuli on low-frequency cells in the cat's inferior colliculus. III. Evidence for cross-correlation. J Neurophysiol 58:562-583.

Zhang DX, Li L, Kelly JB, Wu SH (1998) GABAergic projections from the lateral lemniscus to the inferior colliculus of the rat. Hear Res $117: 1-12$. 